\title{
Recent Advances of Low-Level Light Therapy: Fundamentals, Efficacy and Applications
}

\author{
Jui-Teng Lin* \\ New Vision Inc Taipei, Taiwan \\ *Corresponding author: Jui-Teng Lin, New Vision Inc Taipei, Taiwan
}

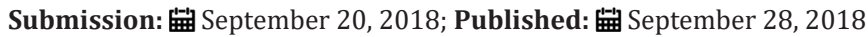

\begin{abstract}
The mechanisms and fundamental of low-level light therapy (LLLT), or photobiomodulation are reviewed and compared with that of photodynamic therapy (PDT). However, LLLT combined with PS will enhance the efficacy, where the generation of singlet oxygen via the PS triplet state is more effective. The biomedical applications of LLLT including dermatology and plastic surgery, wound healings, nerve stimulation, dentistry, cancer therapy, and ophthalmic surgeries are reviewed, where lights (lasers or LED) spectra of UV (200-400) um, visible (400-700) nm, near-IR (700-2900) nm, and mid-IR (3-5) um are presented. Two important laws related to the fundamental and efficacy of the photo-biological systems: The Beer-Lambert law (BLL) and the Bunsen-Roscoe law (BRL) of reciprocity are discussed. LLLT efficacy may be improved by combined with photosensitizer-initiated reactive oxygen species (ROS) for light/heat sensitive ion channels.
\end{abstract}

Keywords: Lasers; Photobiomodulation; Photodynamic therapy; Low level light therapy

\section{Introduction}

Lights (lasers and LEDs) have been used for various medical procedures such as dermatology and cosmetic surgery, wound healings, nerve stimulation, dentistry ophthalmology applications for vision corrections and corneal deceases, and many other therapeutic procedures [1-3]. Combining the nanoparticles, diode lasers have been also used for cancer therapy, bio-sensing, bioimaging, drug delivery, tissue engineering and diagnostics of cancer cell [4-7].

Lights in the near infrared (IR), wavelength of (750-1200) nm, have deeper tissue penetration depths than that of visible lasers. Therefore, near IR lasers are good candidates for procedures which need deep penetrations such as hair removal and nano-gold mediated cancer therapy [1]. On the other hand, visible lasers (430680) $\mathrm{nm}$ with strong absorption in blood and color-dyes have been used for phototherapy of oral cancer, retina deceases and tattoo removal [2]. Mid-IR lasers (1.9-3.0) um and (9.3-10.6) um with strong absorption in water and tissue have been used for super surface procedures or ablative type procedures such as soft and hard tissue ablation. Other IR lasers (1.3-1.6) um have been used for so called minimally invasive procedures such as resurfacing due to their smaller tissue absorption than that of mid-IR lasers $[1,2]$.

High power lights (in CW or pulsed mode) have been reviewed by Lin [1,6-9] for photothermal therapy (PTT) and photodynamic therapy (PDT), using the light spectra of UV (200-400) um, visible (400-700) nm, near-IR (700-2900) nm, and mid-IR (35) um having various penetration depths which define invasive and noninvasive procedures. In contrast to PTT (with high light intensity) and PDT (with photosensitizers), low level light therapy (LLLT), or photobiomodulation (PBM) has a much lower intensity between 5 to $500 \mathrm{~mW} / \mathrm{cm}^{2}$ and does not require photosensitizers (PS). However, LLLT combined with PS will enhance the efficacy, where the generation of singlet oxygen via the PS triplet state is more effective. In this article, we will review the recent progress of LLLT including its fundamental, mechanisms and applications. We will also discuss the efficacy of LLLT with PS and compare the differences and similarity between PDT and LLLT.

\section{The fundamental}

The basic definition and units: Lights are defined by their output "colors", i.e., wavelength (or spectrum) as follows:

UVC (200-300nm), UVB (300-320nm), UVA (320-400) um, visible (400-700) nm, near-IR (700-2900) nm, and mid-IR, (3-5) um, where $1 \mathrm{um}=1,000 \mathrm{~nm}$.

The output of a light is defined by the following units:

Power (P) in $\mathrm{mW}$ (or $\mathrm{W}$ ), $1 \mathrm{~W}=1,000 \mathrm{~mW}$.

Intensity, or irradiance (I) in $\mathrm{mW} / \mathrm{cm}^{2}$ (power per unit area).

Energy (E) in mJ;

Fluence, or dose (F) in $\mathrm{mJ} / \mathrm{cm}^{2}$ (energy density).

Relations: $\mathrm{E}=\mathrm{It}, \mathrm{P}=\mathrm{I} /$ area, $\mathrm{F}=\mathrm{E} /$ area

Laser-matter interaction: Light and tissue (or other media) interaction, in general, could be categorized into three processes: (a) pure thermal, (b) non-thermal (or chemical), and (c) combined 
thermal and non-thermal effects. Lights can be reflected, absorbed, scattered or transparent to the matter. These processes are governed by not only the tissues (media) optical properties but also the laser parameters such as its wavelength, energy, intensity, pulse-width, repetition rate and the operation modes, continuous wave (CW) or pulsed mode. Various fiber structures for effective delivery of the laser energy to the treated areas are also critical in specific applications. Tissue penetration depth (d) of various lasers depends on their wavelength, or their tissue/water absorption coefficients (A), where A is inverse proportional to $d$ (i.e. $d=1 / A$ ). The absorption spectrum in water (or tissue) having 3 major peaks at $1.45 \mathrm{um}, 1.93 \mathrm{um}$ and $2.94 \mathrm{~nm}$, besides the high absorption of $\mathrm{CO}_{2}$ lasers ( 9.6 to $10.6 \mathrm{um})$.

LLLT is essentially a process of photobiomodulation (PBM) without thermal effects, and its does not require photosensitizer (PS), where the light interaction or stimulate biological photoreceptors located inside human cells via electronic transfer from the donors to the acceptors (such as oxygen), augmenting the production of a trans-membrane electric and mechanical proton-H+ gradient. Greater mechanisms of LLLT (and PBM) will be discussed later.

\section{The biomedical applications}

LLLT uses lasers or LEDs typically having power density (or intensity) between 5 to $500 \mathrm{~mW} / \mathrm{cm}^{2}$ and wavelengths of visible (400-700nm), near-infrared (NIR) 780 to $1100 \mathrm{~nm}$; can be a continuous wave (CW) or a pulsed light consisting of a relatively low dose (fluence) ( 0.5 to $50 \mathrm{~J} / \mathrm{cm}^{2}$ ). The wavelength range between 700 and $780 \mathrm{~nm}$ was ineffective as it matches with the absorption spectrum of cytochrome c oxidase. Red (630-670nm) and NIR lights are the most effective due to their larger penetration depth and lower scattering and absorption by tissue chromospheres.

LLLT is also known for biostimulatory and biomodulatory effects in vivo and in vitro and shows not a thermal but photochemical effect by triggering biochemical changes within cells after the application of a lights. The result of LLLT on human tissues is a reduction of inflammation, pain relief, hair growth, adjuvant-induced arthritis, skin and nail psoriasis, breastfeeding, and accelerated tissue regeneration [10-16]. In dentistry, the use of LLLT is to promote wound-healing, dentine repairing process and stimulates the biological activity of mesenchymal stem cells and human periodontal ligament stem cells proliferation [12].

\section{The efficacy and mechanisms}

The mechanisms: The kinetics and mechanism of PDT has been reported by Lin [7-9], in which both oxygen-mediated (type-II) and non-oxygen-mediated (type-I) are presented. LLLT has been known for almost 50 years, since the first reported article using a ruby laser $(694 \mathrm{~nm})$ reported by McGuff et al. [10] (in 1965) for human malignant tumors, and by Mester et al. [11] (in 1968) for hair growth in mice [11]. However, the complete mechanisms of LLLT action remain elusive and was reviewed recently by Ferrares et al. [17] and Freitas \& Hamblin [18]. It was reported [12] that LLLT can augment the oxidative phosphorylation (OXPHOS) modifying the redox basal-status of the whole cell and in particular of mitochondria via electrons transfer from a donor to oxidizing agent, oxygen. The free energy of these reductionoxidation (redox) reactions is spent to synthetize chemical energy of the adenosine triphosphate (ATP). The mechanism of LLLT is based on the absorption of a specific visible red and near-infrared wavelengths by biological photoreceptors located inside human cells, augmenting the production of a trans-membrane electric and mechanical proton- $\mathrm{H}+$ gradient in mitochondria necessary for OXPHOS, and enhancing the activity of mitochondrial complexes IV in an exposure-response relationship [12].

Two major hypothesis about LLLT mechanisms were reported [18]: (i) the photons dissociate nitric oxide from the enzyme causing the increase in electron transport, mitochondrial membrane potential and ATP production, and (ii) ion channels activated by light allowing calcium to enter the cell, The light-activated signaling pathways including the increase of reactive oxygen species (ROS), cyclic adenosine triphosphate, $\mathrm{NO}$ and $\mathrm{Ca}^{2+}$, leading to activation of transcription factors and the increased expression of protein synthesis, cell migration and proliferation, anti-inflammatory signaling, anti-apoptotic proteins, antioxidant enzymes etc. The transcription factors include, such as, nuclear factor kappa B, kappa-B ligand, hypoxia inducible factor, protein kinase B, forkhead box protein M1, peroxisome proliferator-activated receptors, runtrelated transcription factor. The growth factors include, such as, TNF, various interleukins, histamine, TGF- $\beta$, prostaglandins and eicosanoids, brain-derived neurotropic factor, vascular endothelial growth factor, hepatocyte growth factor, basic fibroblast growth factor (bFGF) and keratinocyte growth factor, heat shock protein [18].

LLLT can interact with cells, leading to changes at the molecular, cellular and tissue levels. The tissue mechanism of LLLT include: muscle recovery via the increase of creatine kinase, traumatic brain injury treatment via the newly formed neuroprogenitor cells travelling to the injured region of the cortex, and pain reduction via laser induced analgesia, where a conduction block of central and peripheral nerve fibers and to the release of endorphins. The effects of LLLT on hair growth are already well known, but the exact mechanism still needs clarifying $[12,18]$.

Two possible mechanisms have been proposed by Avci et al. [15]: (i) the small amount of heat produced could induce follicular stem cells to proliferate and differentiate, due to the increased level of heat shock proteins; (ii) the release of certain factors affects the cell cycle and induces angiogenesis. Moreover, LLLT stimulates proliferation and differentiation of osteoblasts, leading to an increased bone formation, accompanied by an increase in the activity of alkaline phosphatase and in osteocalcin expression, as reported by Fujimoto et al. [19]. In contrast to LLLT using low fluence of light, high-fluence low-power laser irradiation (HFLP) activates the mitochondrial apoptosis pathway, altering the cell cycle, inhibiting cell proliferation and even causing cell death. Wu et al. [20] found that HFLP, using light at $633 \mathrm{~nm}$ and $120 \mathrm{~J} / \mathrm{cm}^{2}$, could 
ablate tumors via activation of mitochondrial apoptotic pathway after ROS generation. Cho [21] also observed surviving mediated self-protection of tumor cells against HFLP induced apoptosis, through ROS/cdc25c/CDK1 signaling pathway [22]. Sun et al. [23] reported that signal transducer and activator of transcription 3 (Stat3) was involved in HFLP-induced apoptosis, which is time- and dose-dependent. Steroid receptor co-activator is the main upstream kinase of Stat 3 activation, and the increased ROS generation plays a key role in this process.

The efficacy: The efficacy of LLLT combined with PS has the similar feature as that of type-II PDT, where the increased ROS generation plays a key role in this process. There are two important laws related to the fundamental and efficacy of the photo-biological systems: The Beer-Lambert law (BLL) and the Bunsen-Roscoe law (BRL) of reciprocity $[24,25]$. BLL also known as the Beer-LambertBouguer law is the linear relationship between absorbance and concentration of an absorbing media at various traveling depth. The linearity of BLL is limited by chemical and instrumental factors and the causes of nonlinearity include: deviations in absorption coefficients at high concentrations due to electrostatic interactions between molecules; scattering of light due to particulates in the sample; fluorescence or phosphorescence of the sample; changes in refractive index at high analyte concentration; shifts in chemical equilibria; depletion (breaching) photosensitizer concentration; and non-monochromatic radiation [24]. These nonlinear features and the optimal conditions for maximum efficacy of light-activated biological outcomes have been reported by Lin [26-28]. The optimal conditions could be achieved by, for examples, optimal analytic concentration, light and/or drug dose, light intensity and light penetration depth or analytic concentration profiles [26-28].

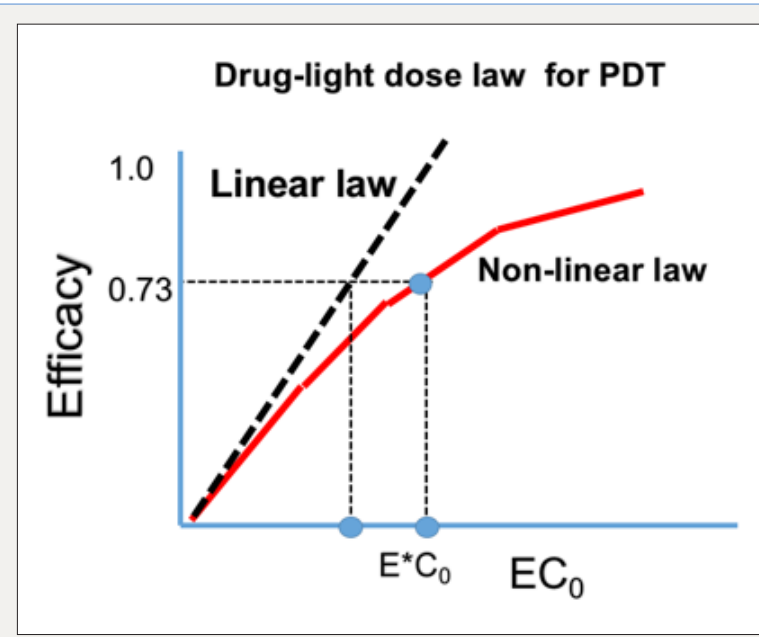

Figure 1: The efficacy versus product [ECO] showing the Bunsen-Roscoe law (BRL, dashed curve) and nonlinear law (solid curve). Also shown are the threshold $\left(\mathrm{E}^{*} \mathrm{CO}\right)$ [27].

BRL of reciprocity [25] stating that the effect of a photo-biological reaction is proportional only to the total irradiation fluence (E=It), or the product of intensity (I) and exposure time (t). To achieve the same efficacy, the required exposure time based on BRL is given by $\mathrm{t}=\mathrm{E} / \mathrm{I}$. Based on BRL, treatment time may be shortened by using a higher intensity while maintaining the similar efficacy. The linear BRL has limitation as that of BLL. A generalized, time dependent Beer-Lambert law (BLL), the non-BRL optimal conditions, and mathematical formulas (scaling laws) have been discussed by Lin [26-28]. Similar to the synergic efficacy of PDT/PTT [6], far infrared light ( 3 to $50 \mathrm{um}$ ) can cause the selective absorption of biological lipid bilayer membranes to enhance the overall LLLT efficacy, via the synergic activation of light/heat sensitive ion channels. Figure 1 shows the PDT efficacy versus light dose (E) and photosensitizer (PS) concentration, $\mathrm{C}_{0}$. Limitation of BRL is shown by the nonlinear law of Lin [28], which demonstrate a higher threshold of [EC $\left.{ }_{0}\right]$ than that of linear BRL.

The efficacy of LLLT combined with PS has the similar efficacy as that of PDT and may be described by a scaling law of a S-function proportional to the light intensity (I) by a power law, $\mathrm{I}^{\mathrm{n}}$, with $0.3<\mathrm{n}<1.2$, depending on the tissues (media) optical properties and the kinetics involved. Clinical data are required to fit the scaling laws. Therefore, variation of exposure time and irradiance may account for conflicting results in the literature. Düzgören et al. [29] recently report the optimal combination of light intensity and dose for maximum efficacy. However, they do not present enough data to show the actual value of $n$ in the scaling law. Lin [27] predicts the $n$ value should be approximately 0.5 to 0.8 for most LLLT procedures. Figure 2 shows an optimal dose $E^{*}$ related to the Arndt-Schulz law having an optimal therapeutic window $[26,27]$. However, without PS, LLLT via the light-activated signaling pathway of reactive oxygen species (ROS), leading to activation of transcription factors is less effective due to the relatively lower rate of generation of ROS. Therefore, LLLT efficacy may be improved by combined with PTT (using light-induced temperature) and PS-initiated ROS for light/ heat sensitive ion channels.

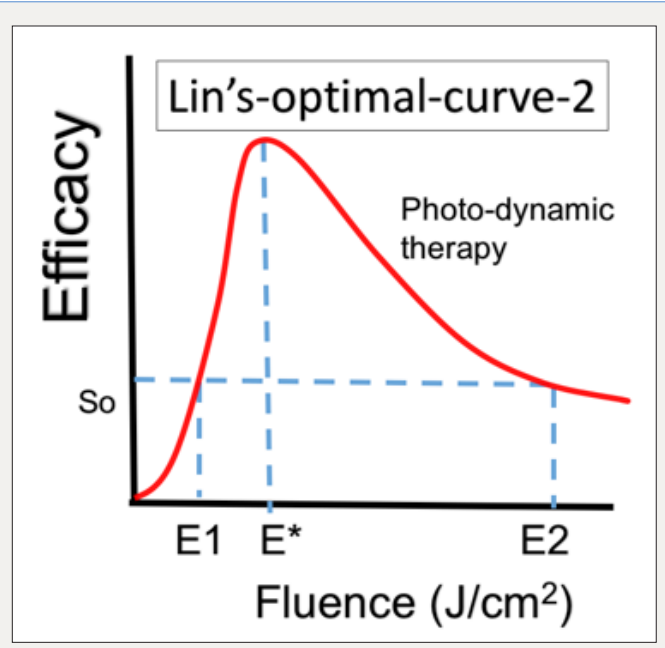

Figure 2: Optimal dose $E^{*}$ related to the Arndt-Schulz law showing an optimal therapeutic window, (E1 and E2), providing a safe and effective therapy [27].

The role of light dose has been reported by Lin [30], Alkin et al. [31] \& Dhiran et al. [32]. Effort to minimize these complications, various modified PDT protocols have been explored involving reduced verteporfin dosage, laser fluence, or a combination of both. 
Half-fluence, half-dose, half-fluence and micropulse, 1/3 dose, and minimal-fluence protocols have all demonstrated some degree of treatment effect. Half-dose verteporfin and half-fluence treatments are the two most described modified protocols [31,32].

\section{Conclusion}

We have presented the limitation of the linear BRL and BLL. A generalized, time dependent BLL provides the non-BRL optimal conditions. The optimal dose is related to the Arndt-Schulz law having an optimal therapeutic window. Without PS, LLLT via the light-activated signaling pathway of reactive oxygen species (ROS), leading to activation of transcription factors is less effective due to the relatively lower rate of generation of ROS. Therefore, LLLT efficacy may be improved by combined with PTT (using lightinduced temperature) and PS-initiated ROS for light/heat sensitive ion channels.

\section{References}

1. Lin JT (2016) Progress of medical lasers: Fundamentals and applications. Medical Device Diagn Eng 1: 36-41.

2. Lin JT (2017) Design aspects of medical laser devices. Medical Device Diagn Eng 2: 75-77.

3. Lin JT (2017) Progress of the 30-year laser vision technology. J Ophthalmol Clinical Res 3: 1-4.

4. Lin JT, Chen KT, Liu HW (2017) Progress of nanotechnology for phototherapy: Fundamentals and applications. Med Devices Diagn Eng, doi: 10.15761/MDDE.1000124.

5. Lin JT, Chen KT, HW Liu (2018) Analysis of kinetics and efficacy of anti-cancer via oxygen-enhanced photodynamic therapy. J Cancer Research update 7: 21-23.

6. Lin JT, Chen KT, Liu HW (2018) Novel techniques for improving anti-cancer efficacy via synergistic phototherapy. Op Acc J Bio Eng \& Biosc 2(1): 124-128.

7. Lin JT (2018) Kinetics and efficacy of photo-activated crosslink for tissue-engineering. Op Acc J Bio Eng \& Biosc 2(4): 214-218.

8. Lin JT (2018) Efficacy S-formula and kinetics of oxygen-mediated (typeII) and non-oxygen-mediated (type-I) corneal cross-linking. Ophthalmology Research 8(1): 1-11.

9. Lin JT (2018) A critical review on the kinetics, efficacy, safety, nonlinear law and optimal protocols of corneal cross-linking. J Ophthalmology \& Visual Neuroscinece 3(1): 017.

10. McGuff PE, Deterling RA, Gottlieb LS (1965) Tumoricidal effect of laser energy on experimental and human malignant tumors. N Engl J Med 273(9): 490-492

11. Mester E, Szende B, Gärtner P (1968) The effect of laser beams on the growth of hair in mice. Radiobiol Radiother (Berl) 9(5): 621-626.

12. Vincenzo G, Maddalena V, Fabrizia D (2018) Analysis of changes induced in human periodontal ligament, dental pulp, bone marrow and adipose stem cells by low level laser therapy. A review and new perspectives. Biomed J Sci \&Tech Res 4(2): 1-7.

13. Maranda EL, Nguyen AH, Lim VM (2016) Laser and light therapies for the treatment of nail psoriasis. J Eur Acad Dermatol Venereol 30(8): 1278-1284

14. Hsieh YL, Cheng YJ, Huang FC (2014) The fluence effects of low-level laser therapy on inflammation, fibroblast-like synoviocytes, and synovial apoptosis in rats with adjuvant-induced arthritis. Photomed Laser Surg 32(12): 669-677.

15. Avci P, Gupta GK, Clark J (2014) Low-level laser (light) therapy (LLLT) for treatment of hair loss. Lasers Surg Med 46(2): 144-151.

16. Buck ML, Eckereder G, Amir LH (2016) Low level laser therapy for breastfeeding problems. Breastfeed Rev 24(2): 27-31.

17. Ferrares C, Kaippert B, Avci P (2015) Low-level laser (light) therapy increases mitochondrial membrane potential and ATP synthesis in C2C12 myotubes with a peak response at 3-6 hours. Photochem Phtobiol 91(2): 411-416.

18. Freitas de Freitas L, Hamblin MR (2016) Proposed mechanisms of photobiomodulation or low-level light therapy. IEEE J Sel Top Quantum Electron 22(3), doi:10.1109/JSTQE.2561201.

19. Fujimoto K, Kiyosaki T, Mitsui N (2010) Low-intensity laser irradiation stimulates mineralization via increased BMPs in MC3T3-E1 cells. Lasers Surg Med 42(6): 519-526.

20. Wu S, Zhou F, Wei Y, Chen WR (2014) Cancer phototherapy via selective photoinactivation of respiratory chain oxidase to trigger a fatal superoxide anion burst. Antioxid Redox Signal 20(5): 733-746.

21. Choi M, Kim JE, Cho KH, Lee JH (2013) In vivo and in vitro analysis of low-level light therapy: A useful therapeutic approach for sensitive skin. Lasers Med Sci 28(6): 1573-1579.

22. Chu J, Wu S, Xing D (2010) Survivin mediates self-protection through ROS/cdc25c/CDK1 signaling pathway during tumor cell apoptosis induced by high fluence low-power laser irradiation. Cancer Lett 297(2): 207-219.

23. Sun X, Wu S, Xing D (2010) The reactive oxygen species-Src-Stat3 pathway provokes negative feedback inhibition of apoptosis induced by high-fluence low-power laser irradiation. FEBS J 277(22): 4789-4802.

24. Lambert JH (1760) Photometry, or, on the measure and gradations of light, colors, and shade. Eberhardt Klett, Germany.

25. Bunsen RW, Roscoe HE (1862) Photochemical researches-Part V. On the measurement of the chemical action of direct and diffuse sunlight. Proc R Soc Lond 12: 306-312.

26. Lin JT (2018) Lin- Scaling-laws for optimal efficacy in photo-biological systems versus Arndt-Schulz-Law and Bunsen-Roscoe law. Med Devices Diagn Eng 3: 120-123.

27. Lin JT (2018) Optimal efficacy in light-activated biomedical systems and nonlinear laws versus linear Beer-Lambert law and Bunsen- Roscoe reciprocal law. Op Acc J Bio Eng \& Biosc 1(5): 114-117.

28. Lin JT (2018) Efficacy S-formula and kinetics of oxygen-mediated (typeII) and non-oxygen-mediated (type-I) corneal cross-linking. Ophthalmology Research 8(1): 1-11.

29. Düzgören I, Ruhi MK, Gulsoy M (2017) Effect of different laser power densities on photobiomodulation of L929 cell line.

30. Lin JT (2018) Analysis of drug-light dose on the efficacy of photodynamic therapy of age related macular degeneration. J Ophthalm Studies 1(1): $1-4$.

31. Alkin Z, Perente I, Ozkaya A (2014) Comparison of efficacy between low-fluence and half-dose verteporfin photodynamic therapy for chronic central serous chorioretinopathy. Clin Ophthalmol 8: 685-690.

32. Dhiran NA, Yang Y, Somani S (2017) Long-term outcomes in half-dose verteporfin photodynamic therapy for chronic central serous retinopathy. Clinical Ophthalmology 11: 2145-2149. 
(c) (i) Creative Commons Attribution 4.0 International License

For possible submissions Click Here

Submit Article

$\begin{array}{ll}\text { RMES } & \begin{array}{l}\text { Researchin } \\ \text { Medicil\& } \\ \text { Engieering } \\ \text { Sciences }\end{array} \\ \text { Benefits of Publishing with us }\end{array}$

